\title{
Isolation Enhancement Between Two Packed Antennas With Coupling Element
}

\author{
Jiunn-Nan Hwang and Shyh-Jong Chung
}

\begin{abstract}
This letter introduces a coupling element to enhance the isolation between two closely packed antennas for $2.4-\mathrm{GHz}$ wireless local area network (WLAN) application. The proposed structure occupies two antenna elements and a coupling element in between. By putting a coupling element that artificially creates an additional coupling path between the antenna elements, the antenna isolation can be enhanced. The advantage of this design is that no extra space is needed for antenna elements. With the proposed design, more than $15 \mathrm{~dB}$ isolation can be achieved for two parallel individual inverted-F antennas (IFAs) with $5-\mathrm{mm}$ spacing. Parametric studies for the design are also included to show how to increase isolation bandwidth and control the isolation frequency.
\end{abstract}

Index Terms-Coupling element, inverted-F antennas (IFAs), isolation, wireless local area network (WLAN).

\section{INTRODUCTION}

$\mathbf{T}$ HE ISOLATION between antennas is a critical parameter in many practical applications such as antenna arrays, diversity antennas, and also multiple-input-multiple-output (MIMO) communication systems. However, when antennas are closely packed, strong mutual coupling will degrade radiation patterns and decrease antenna efficiency, which will cause deterioration in signal-to-noise ratio and signal to-interference-plus-noise ratio of the systems [1]. For planar inverted-F antennas (PIFAs) oriented either collinear, orthogonal, or parallel above a single ground plane with air substrate, it is often stated that the interelement spacing should be at least greater than half of the free space in order to achieve high isolation [2]. Many papers have been presented for isolation enhancement between packed antennas. For example, mushroom-like electromagnetic band-gap (EBG) structures can suppress the surface wave between antenna elements [3] and thus reduce the mutual coupling between antenna elements. In [4], the method of using lumped circuit networks on the input ports is proposed to decouple two closely spaced PIFAs. With the decoupling circuit networks, the isolation between the two PIFAs can be improved up to $30 \mathrm{~dB}$. In [5], a suspended neutralization strip is inserted and physically connected to the antenna elements for improving isolation. This line samples a certain amount of the signal on one antenna element and

Manuscript received July 24, 2011; revised September 25, 2011; accepted October 19, 2011. Date of publication November 04, 2011; date of current version November 28, 2011.

The authors are with the Department of Communication Engineering, National Chiao Tung University, Hsinchu 300, Taiwan (e-mail: sjchung@cm.nctu. edu.tw).

Color versions of one or more of the figures in this letter are available online at http://ieeexplore.ieee.org.

Digital Object Identifier 10.1109/LAWP.2011.2174957 delivers to the other antenna element in order to cancel out the existing mutual coupling. In [6], a novel way to achieve neutralization of the antenna through folded lines connected to a printed circuit board (PCB) was proposed for antenna isolation. In [7] and [8], a coupling element is introduced to improve the antenna isolation. However, extra space is needed for this design. In [9], the embedded strip resonator between two PIFAs is used for antenna isolation improvement. However, the proposed design occupied a large space between the antennas.

In this letter, we propose a new coupling element between the antennas in order to create an additional coupling path for enhancing the isolation. The coupling element is placed between antennas and therefore no extra space is needed with this design. This coupling element is not physically connected to the antenna elements and is flexible for controlling the center frequency, bandwidth, and level of isolation. To demonstrate the idea, two antenna elements for using in $2.4 \mathrm{GHz}$ WLAN band are studied. From this study, it is found that the design can achieve more than $15 \mathrm{~dB}$ isolation improvement with $5 \mathrm{~mm}$ antenna spacing. The detail parametric studies are provided, which show the design of the proposed structure.

\section{ANTENNA Design}

The details of our proposed design are shown in Fig. 1. It can be seen that two identical antenna elements operated at $2.4 \mathrm{GHz}$ are integrated on a low-cost FR4 substrate with a PCB thickness of $1.6 \mathrm{~mm}$ and relative permittivity is 4.3 . Their edge-to-edge separation is just $5 \mathrm{~mm}\left(0.04 \lambda_{0}\right)$. The antennas are located on the top left-hand side and right-hand side of the PCB, respectively, and the resonant length is approximately a quarter-wavelength. The coupling element is introduced for enhancing isolation. It is located between the two antenna elements and occupies the dimension of $5 \times 13 \mathrm{~mm}^{2}$. The proposed coupling element is formed by a coupling pad and a thin wire connected to backside ground. The advantage of this design is that no extra space is needed for antenna elements. The concept for isolation enhancement with this design is discussed briefly.

Assume the excited current is fed into Antenna 1. Since Antenna 2 is placed very close to Antenna 1, the strong coupled current on Antenna 2 is approximately $180^{\circ}$ out of phase with the excited current. The proposed coupling element is placed between the two antennas. In this condition, the coupling starts from Antenna 1, through to the coupling element, which, in turn, couples to Antenna 2. The coupled current on coupling element is approximately in phase with excited current on Antenna 1. If the coupling element in this path is adjusted properly, the two coupled currents can be canceled out so that the overall resultant coupled current is canceled. The addition 


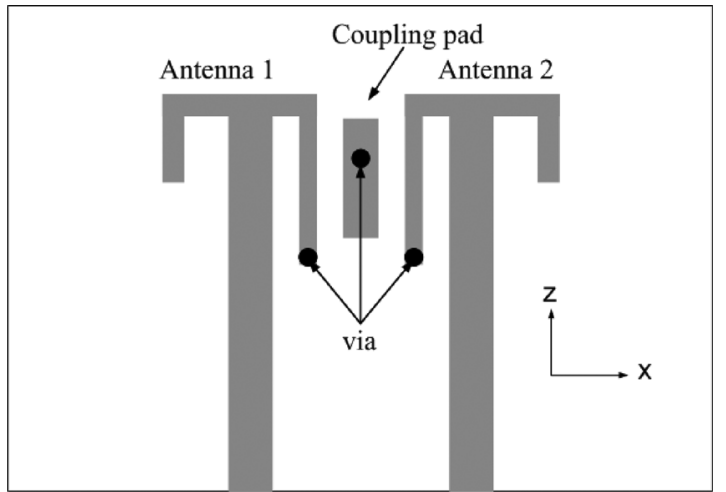

(a)

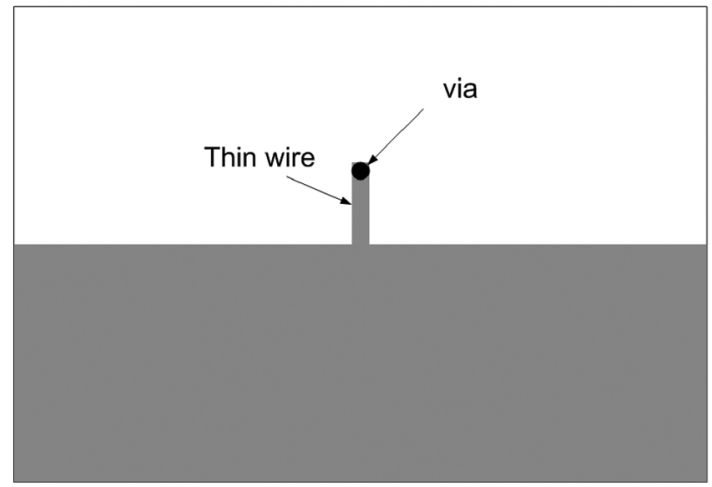

(b)

Fig. 1. Geometry of two antennas using coupling element for isolation enhancement. (a) Front side. (b) Back side.

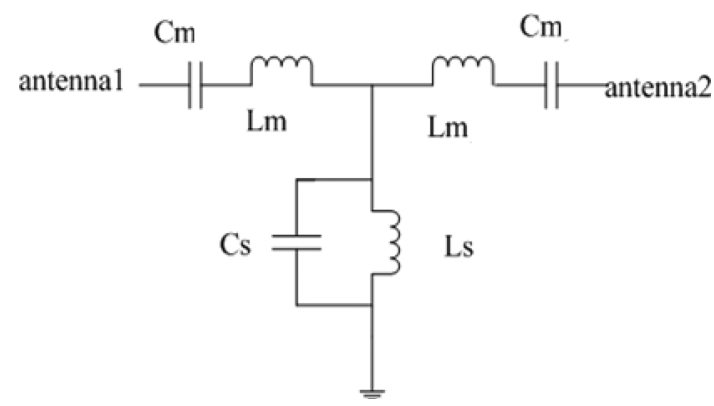

Fig. 2. Equivalent circuit model of the coupling element.

of the coupling element will also affect the self-impedance of the antennas, and this effect can be handled by adjusting the antenna matching appropriately.

\section{Parametric Coupling Element Study}

We have performed the parametric studies, and they explain how to control the center frequency, bandwidth, and level of isolation. The coupling element structure will significantly affect isolation improvement. The circuit model of the proposed design is shown in Fig. 2. The $L_{\mathrm{s}}$ and $C_{\mathrm{s}}$ are the ground inductance and self-capacitance of the coupling element. The $L_{\mathrm{m}}$ and $C_{\mathrm{m}}$ represent the mutual inductance and mutual capacitance between the antenna and the coupling element. The parameters of the equivalent circuit are studied. The capacitance $C_{\mathrm{m}}$ and ground inductance $L_{\mathrm{s}}$ will significantly affect the isolation level

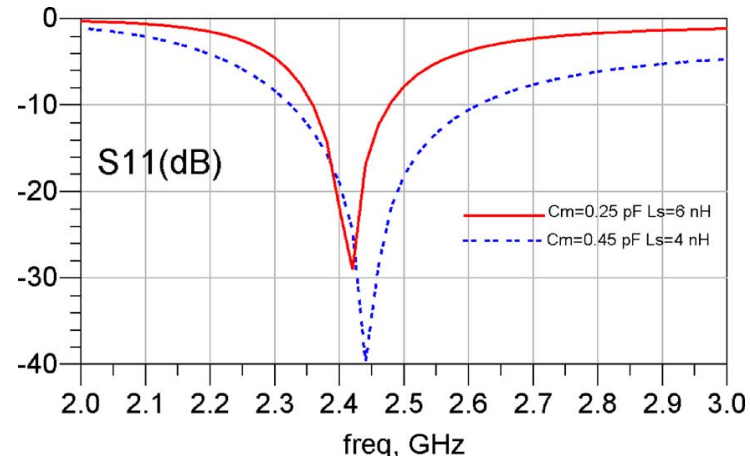

Fig. 3. Simulated $S$-parameters for equivalent circuit model.

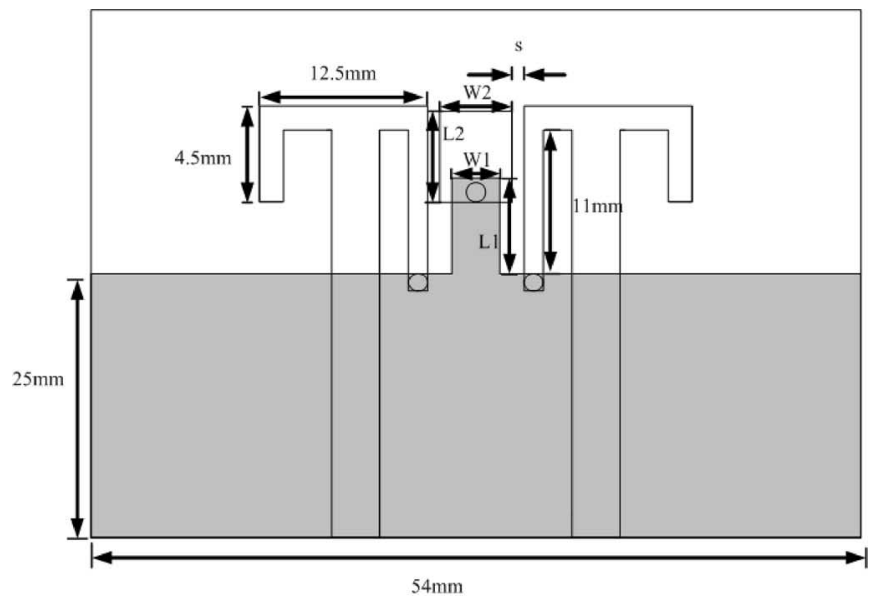

(a)

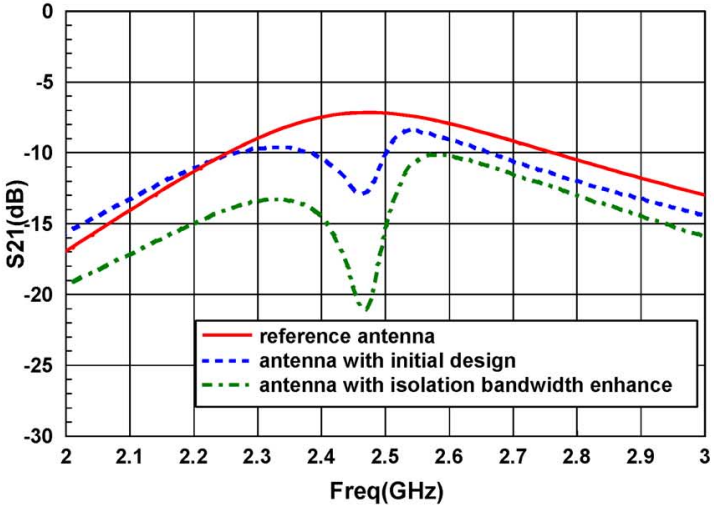

(b)

Fig. 4. (a) Proposed design for isolation bandwidth enhancement. (b) Simulated $S$-parameters for the proposed design.

and bandwidth. In this letter, $L_{\mathrm{m}}$ and $C_{\mathrm{s}}$ are extracted from the Ansoft Q3D Extractor, and they are $1.5 \mathrm{nH}$ and $0.2 \mathrm{pF}$, respectively. Similar $L_{\mathrm{m}}$ and $C_{\mathrm{s}}$ values also can be obtained from analytical formula. First, the $C_{\mathrm{m}}=0.25 \mathrm{pF}$ and $L_{\mathrm{s}}=6 \mathrm{nH}$ are used for operation at $2.4-\mathrm{GHz}$ band. The simulated $S_{11}$ based on the Agilent ADS commercial tool is shown in Fig. 3. The increase of the mutual capacitance and reduction of the ground inductance are considered. The $C_{\mathrm{m}}$ and $L_{\mathrm{s}}$ are changed to be $0.45 \mathrm{pF}$ and $4 \mathrm{nH}$, respectively. From this circuit model, it is found that the bandwidth of the coupling element can be improved with increasing the mutual capacitance. 

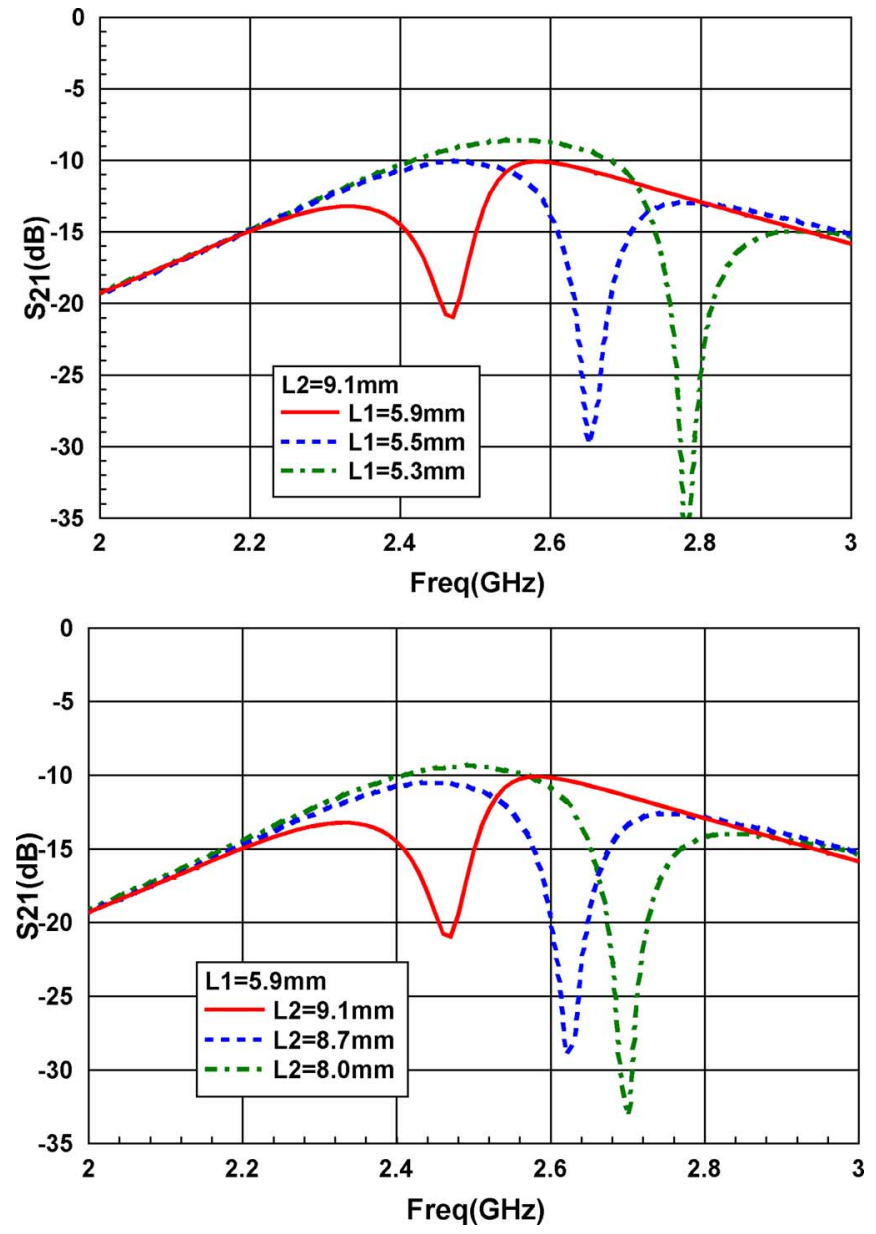

Fig. 5. Parametric study of the coupling element.

Increasing the bandwidth of the coupling element will also increase the isolation level. The reason is that increase the capacitance will increase the field strength of the coupling element, which cancels out the mutual coupling between antenna elements and thus enhances the isolation. To verify the effect of isolation bandwidth improvement, the antenna structure of Fig. 4 is studied. By controlling the size of the coupling pad and thin wire, the coupling element can be operated at $2.4-\mathrm{GHz}$ WLAN band. The detailed geometry of the coupling element is shown in Fig. 4. For the initial design, the geometry parameters $L_{1}=7 \mathrm{~mm}, W_{1}=1 \mathrm{~mm}, L_{2}=9.5 \mathrm{~mm}$, and $W_{2}=2.6 \mathrm{~mm}$. The corresponding gap $s$ between the coupling element and antenna is $1.2 \mathrm{~mm}$. The coupling element is then modified to increase the capacitance between the antenna and coupling element. The width of the ground wire $W_{1}$ is also increased to reduce the ground inductance for operation at $2.4 \mathrm{GHz}$. The proposed design with $L_{1}=5.9 \mathrm{~mm}, W_{1}=4 \mathrm{~mm}, L_{2}=9.1 \mathrm{~mm}$, and $W_{2}=4.5 \mathrm{~mm}$ is studied. The gap $s$ is changed to be $0.25 \mathrm{~mm}$. The simulated isolation for the proposed design is shown in Fig. 4. The results are compared to reference antenna elements without coupling element. From the simulation results, it is found the bandwidth of this design can be significantly improved and the isolation can be above $15 \mathrm{~dB}$ for $2.4-\mathrm{GHz}$ WLAN band.

The parametric study is based on the geometry given in Fig. 4. Referring to Fig. 4, the coupling element has two key param-

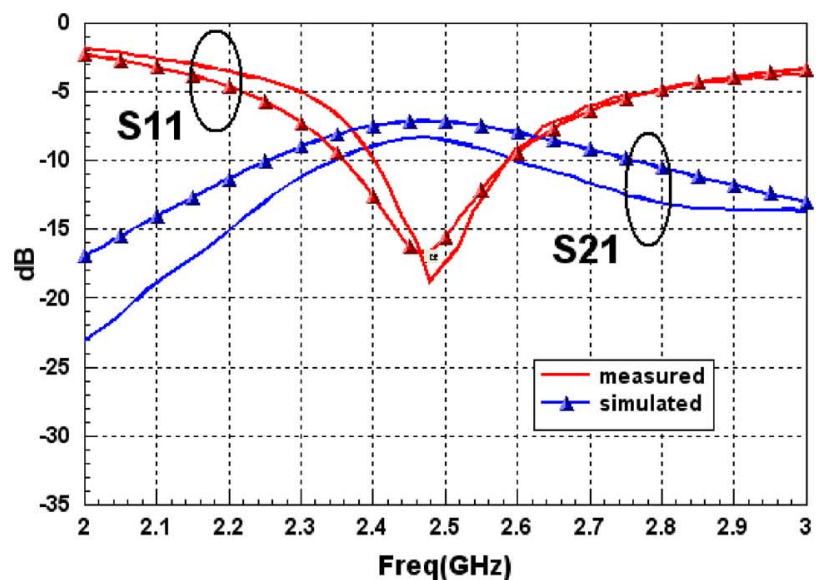

(a)

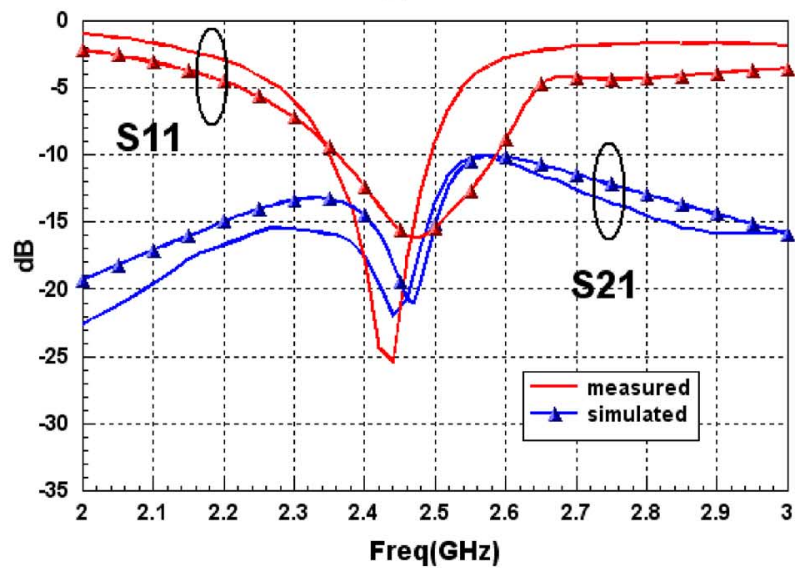

(b)

Fig. 6. (a) Measured $S$-parameters for the reference antenna elements. (b) Measured $S$-parameters of the proposed design with isolation enhancement.

eters, which we refer to the $L_{1}$ and $L_{2}$ as labeled in Fig. 4. We simulate the proposed structure in Fig. 4 and vary these two parameters to understand how to control the structure. For the studies on the effect of $L_{1}$, first we fix the value $L_{2}$ and vary the $L_{1}$ from 5.3 to $5.9 \mathrm{~mm}$. Increasing $L_{1}$ will increase the parasitic inductance. In Fig. 5, we can observe that the position of the maximum isolation shifts to a lower frequency when the $L_{1}$ increases. Second, we fix the value of $L_{1}$ and vary $L_{2}$ from 9.1 to $8.0 \mathrm{~mm}$. The parasitic capacitance between the coupling element and antennas will be decreased after decreasing the $L_{2}$ length. In Fig. 5, we can observe that the position of the maximum isolation shifts to higher frequency when the $L_{2}$ decreases. From the result, we can notice that both the $L_{1}$ and $L_{2}$ have a significant effect on the position of maximum isolation. It is found that they control the resonant length of the coupling element and shift the position of maximum isolation in the frequency range.

\section{MEASUREMENT RESUlts}

The simulated and measured results are shown in Fig. 6. The isolation between the two antennas is $8 \mathrm{~dB}$ at $2.45 \mathrm{GHz}$ for the reference antenna without coupling element. With the proposed coupling element occupied between the antennas, a maximum isolation above $22 \mathrm{~dB}$ for $2.4-\mathrm{GHz}$ WLAN band can be obtained. Although there is a slight frequency shift of the $S_{11}$, 


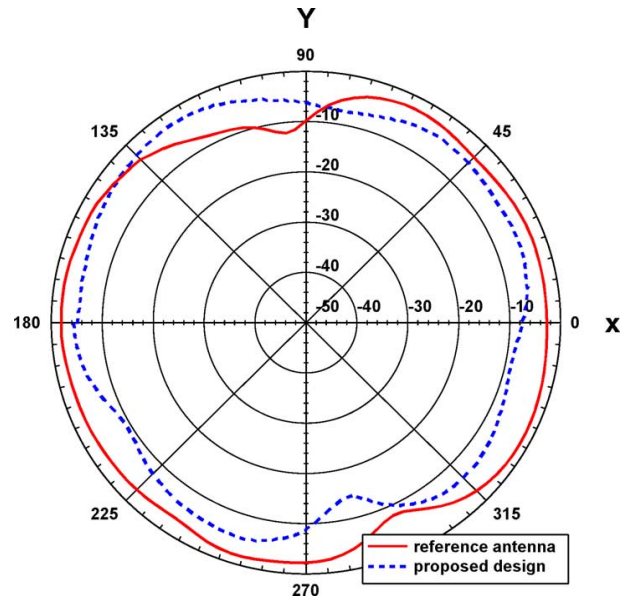

Fig. 7. Measured radiation pattern comparison for reference antenna element and proposed design.

this effect can be compensated by modifying the antenna length. According to the measurement result, it shows that the antenna isolation can be above $15 \mathrm{~dB}$ from 2.4 to $2.5 \mathrm{GHz}$.

To further confirm the performance of the design, the measured antenna gain pattern is also carried out. The antenna patterns for exciting Port 1 (left port) at $2.4 \mathrm{GHz}$ are shown in Fig. 7. The peak gain for the reference antenna and the proposed design are -1.93 and $-2.5 \mathrm{~dB}$, respectively. Although the antenna pattern will be somewhat affected with the additional coupling element, the maximum antenna gain can be less affected with the proposed design. The radiation efficiency of the proposed antenna is $79 \%$ from simulation, and this design can be useful for practical internal laptop antenna application.

\section{CONCLUSION}

In this letter, a coupling element to enhance isolation for closely packed antennas operating at $2.4-\mathrm{GHz}$ WLAN band is proposed. We artificially create an additional coupling path by utilizing a coupling element to enhance the coupling between the antenna elements. The concept of improving isolation for the proposed coupling element is discussed. The antenna isolation above $15 \mathrm{~dB}$ can be achieved with their spacing just $5 \mathrm{~mm}$. The parameters of the proposed coupling element are evaluated to control the frequency band of the maximum isolation, peak isolation, and the bandwidth of the transmission reduction.

\section{REFERENCES}

[1] J. W. Wallace, M. A. Jensen, A. L. Swindlehurst, and B. D. Jeffs, "Experimental characterization of the MIMO wireless channel: Data acquisition and analysis," IEEE Trans. Wireless Commun., vol. 2, no. 2, pp. 335-343, Mar. 2003.

[2] E. M. Friel and K. M. Pasala, "Effects of mutual coupling on the performance of STAP antenna arrays," IEEE Trans. Aerosp. Electron. Syst., vol. 36, no. 2, pp. 518-527, Apr. 2000.

[3] D. Sievenpiper, L. Zhang, R. F. J. Broas, N. G. Alexopolous, and E. Yablonovitch, "High-impedance electromagnetic surfaces with a forbidden frequency band," IEEE Trans. Microw. Theory Tech., vol. 47, no. 11, pp. 2059-2074, Nov. 1999.

[4] S.-C. Chen, Y.-S. Wang, and S.-J. Chung, "A decoupling technique for increasing the port isolation between two strongly coupled antennas," IEEE Trans. Antennas Propag., vol. 56, no. 12, pp. 3650-3658, Dec. 2008.

[5] A. Diallo, C. Luxey, P. Le Thuc, R. Staraj, and G. Kossiavas, "Study and reduction of the mutual coupling between two mobile phone PIFAs operating in the DCS1800 and UMTS bands," IEEE Trans. Antennas Propag., vol. 54, no. 11, pt. 1, pp. 3063-3074, Nov. 2006.

[6] A. Chebihi, C. Luxey, A. Diallo, P. Le Thuc, and R. Staraj, "A novel isolation techniques for closely spaced PIFAs for UMTS mobile phones," IEEE Antennas Wireless Propag. Lett., vol. 7, pp. 665-668, 2008.

[7] C.-H. Lee, S.-Y. Chen, and P. Hsu, "Integrated dual planar inverted-F antenna with enhanced isolation," IEEE Antennas Wireless Propag. Lett., vol. 8, pp. 963-965, 2009.

[8] A. C. K. Mak, C. R. Rowell, and R. D. Murch, "Isolation enhancement between two loosely packed antennas," IEEE Trans. Antennas Propag., vol. 56, no. 11, pp. 3411-3419, Nov. 2008.

[9] T.-W. Kang and K.-L. Wong, "Isolation improvement of 2.4/5.2/5.8 $\mathrm{GHz}$ WLAN internal laptop computer antennas using dual-band strip resonator as a wavetrap," Microw. Opt. Technol. Lett., vol. 52, pp. 58-64, Jan. 2010. 